transitions, and so it is interesting that a CDK inhibitor localizes with a protein that potentially both suppresses apoptosis and regulates a cell-cycle checkpoint. These observations, however, are based largely on immunofluorescence microscopy, and these proteins have not yet been shown to co-exist in a multiprotein complex. Interestingly, p21 deficiency which is known to promote inappropriate centrosome duplication and aneuploidy ${ }^{14}$ exacerbated the cell-division defects caused by suppression of survivin ${ }^{1}$. Whether or not p21 and survivin directly collaborate in cytokinesis is far from clear, however, and reports that p21 is a substrate of caspases may be entirely unrelated to these observations.

Although an interesting protein with clear relevance to cancer therapy, myriad questions about survivin remain unanswered. Fundamental to understanding survivin's function is the issue of whether it suppresses apoptosis only during or immediately after mitosis, when the protein is localized to mitotic spindles and midbodies of dividing cells. What is the role of the cytosolic pool of survivin seen in tumours in immunostaining experiments ${ }^{2}$ ? Studies using synchronized populations of cells, and time-lapse photographic observation of individual cells treated with survivin inhibitors, would be enlightening. Also, what proteins within mitotic spindles does survivin bind to directly, and exactly how does it participate in cell-division control? Genetic analysis of analogous BIR-domain proteins in yeast might provide some clues. Might survivin, when bound to centrosomes, indeed be required to suppress a default apoptosis pathway that should not be activated when the spindle is assembled properly? To answer this, it would be useful to know whether caspases are usually activated during mitosis, and whether biologically active survivin is required to bind and inhibit these proteases. Analysis of the structure of the BIR domain of survivin alone and in a complex with a caspase would be informative here, particularly given recent reports that the BIR2 domain of XIAP and the BIR3 domain of cIAP1 possess distinctly different folds, and that segments outside the BIR domains of IAPs may be essential for caspase suppression ${ }^{15,16}$.

Regardless of the answers, BIR-domain proteins have brought the worlds of cell-cycle and cell-death research into collision. These zinc-binding proteins possess an ancient function relevant to cell division in lower organisms that may have been modified during evolution to serve other purposes, such as caspase suppression and possibly receptormediated signal transduction ${ }^{4,5}$. In the case of survivin, time will tell whether these independent functions of cell-cycle and apoptosis regulation are inextricably linked, thereby creating a unique and important cell-division checkpoint that ensures the apoptotic demise of genetically unstable cells.

John C. Reed is at The Burnham Institute, La Jolla, California 92037, USA.

e-mail: jreed@burnham.org

Steven I. Reed is at the Scripps Research Institute, La Jolla, California 92077, USA.

e-mail: sreed@scripps.edu

1. Li, F. et al. Nature Cell Biol. 1, 461-466 (1999).

2. Ambrosini, G., Adida, C. \& Altieri, D. Nature Med. 3, 917-921 (1997).

3. Li, F. et al. Nature $396,580-587$ (1998)

4. Miller, L. Trends Cell Biol. 9, 323-328 (1999).

5. Deveraux, Q. \& Reed, J. Genes Dev. 13, 239-252 (1999).

6. Ambrosini, G., Adida, C., Sirugo, G. \& Altieri, D. C. J. Biol. Chem. 273, 11177-11182 (1998).

7. Tamm, I. et al. Cancer Res. 58, 5315-5320 (1998).

8. Kobayashi, K., Hatano, M., Otaki, M., Ogasawara, T. \& Tokuhisa, T. Dev. Biol. 96, 1457-1462 (1999).

9. Takahashi, R. et al. J. Biol. Chem. 273, 7787-7790 (1998).

10. Deveraux, Q. et al. EMBO J. 18, 5242-5251 (1999).

11. Fraser, A., James, C., Evan, G. \& Hengartner, M. Curr. Biol. 9 , 292-301 (1999)

12. Uren, A. G., Coulson, E. J. \& Vaux, D. L. Trends Biochem. Sci. 23, 159-162 (1998)

13. Vaux, D. et al. Proc. Natl Acad. Sci. Cell Biol. 96, 10170-10175 (1999).

14. Winey, M. Curr. Biol. 9, R449-R452 (1999).

15. Sun, C. et al. Nature 401, 818-821 (1999).

16. Hinds, M., Norton, R., Vaux, D. \& Day, C. Nature Struct. Biol. 6, 648-651 (1999)

\title{
A new piece in the telomere jigsaw
}
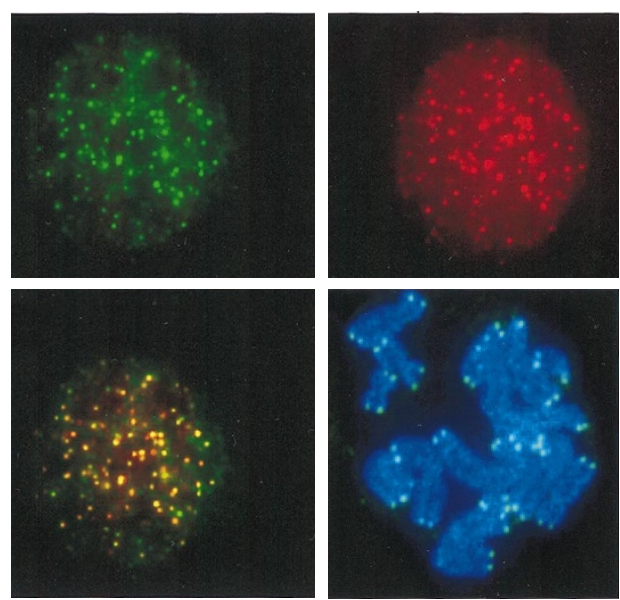

One feature of many human cancers, as well as of human germ lines and early embryonic cells, is that they express an enzyme called telomerase. This enzyme's job is to add DNA to telomeres - the specialized DNA-protein structures that cap and protect the ends of chromosomes. Without telomerase, the DNA part of the telomeres shortens every time a cell multiplies, because each time the cell copies its chromosomes it fails to replicate part of the telomere ends. Telomeres shorten like this in normal adult human somatic cells, which don't express telomerase. Eventually, these cells can no longer safely duplicate their chromosomes and so stop dividing. This 'senescence' is one of the possible causes of cellular ageing, and the fact that some cancer cells express telomerase is one reason why they can keep on multiplying where normal cells cannot

In cells that express telomerase, other proteins are needed to restrict its activity so that telomeres don't get too long. Two such proteins are TRF1 and TRF2, which bind telomeric DNA. TIN2 discovered by Kim et al. (Nature Genet. 23, 405-412; 1999) — is another. Kim et al. show that TIN2 localizes to telomeres in dividing human cells, as seen here (bottom right; pale blue identifies TIN2 and dark blue identifies DNA). It can't bind to telomeres itself, and is probably recruited by TRF1: TIN2 binds to TRF1 in vitro and in human cell lines, and TIN2 and TRF1 co-localize (anticlockwise from top right to bottom left: TRF1 staining, TIN2 staining and a merged image). TIN2 can restrict telomerase's telomere-extending activity, and its amino terminus is important for this: in a telomeraseexpressing human cell line, a mutant TIN2 protein that lacks part of its amino terminus results in greatly expanded telomeres.

Somehow, these proteins may work together to restrict telomerase's activity. Kim et al. speculate that TIN2 may recruit telomerase inhibitors to telomeres by means of its amino terminus. Or maybe TIN2 promotes the formation of a telomeric structure that prevents telomerase from getting to its substrate - the unpaired DNA at the chromosome's 3 ' end. A host of new experiments aimed at working out TIN2's structure and function is certain to be sparked by these results, and we will no doubt learn much about the complex events of cell ageing and cancer biology in the process.

AMANDA TROMANS 